\title{
A etnofarmacologia como terapia complementar na atenção básica: uma revisão
}

\section{integrativa}

\author{
Ethnopharmacology as a complementary therapy in primary care: an integrative review \\ La etnofarmacología como terapia complementaria en atención primaria: una revisión integradora
}

Recebido: 08/10/2021 | Revisado: 14/10/2021 | Aceito: 15/10/2021 | Publicado: 18/10/2021

\author{
Maria Clara Barradas Leão \\ ORCID: https://orcid.org/0000-0002-3554-4185 \\ Centro Universitário Santo Agostinho, Brasil \\ E-mail: mariaclaraleao22@gmaial.com \\ Yanka Delmondes Campelo \\ ORCID: https://orcid.org/0000-0002-4664-6240 \\ Centro Universitário Santo Agostinho, Brasil \\ E-mail: yankadelmondes@outlook.com \\ Luciane Lima da Silva \\ ORCID: https://orcid.org/0000-0002-2391-4812 \\ Centro Universitário Santo Agostinho, Brasil \\ E-mail: lucianenppm@yahoo.com.br
}

\begin{abstract}
Resumo
A etnofarmacologia trata do conhecimento popular referente aos tradicionais sistemas da medicina, ela consiste em informações adquiridas ao longo da vida pela população usuária da flora medicinal com o estudo farmacológico. Objetivou-se analisar as evidências científicas sobre o conhecimento dos profissionais de saúde acerca do uso da etnofarmacologia como terapia complementar na atenção básica. Para isso realizou-se uma revisão integrativa de literatura, por meio das plataformas online de pesquisa Us national Library of Medicine (PUBMED) e Biblioteca Virtual em Saúde (BVS) nas bases de dados LILACS, MEDLINE E BDENF. Para a construção da questão de pesquisa da revisão integrativa, utilizouse a estratégia Pico, essencial para a aumentar da especificidade e clareza conceitual da situação a ser estudada, fornecendo resultados de maior qualidade e singularidade. Foram selecionados artigos indexados em periódicos nacionais e internacionais, fornecidos na íntegra, e estudos encontrados nos últimos 5 anos nos idiomas português, inglês e espanhol. Os resultados sugerem que ainda que a fitoterapia seja uma prática benéfica para muitos, ainda há fatores que agem como barreiras para a consolidação dessa terapêutica dentro dos serviços de saúde. Por isso, torna-se sumamente importante a mobilização de estratégias que possam abrir caminhos para a implementação eficaz das práticas etnofarmacologicas como parte integrante do manejo da saúde dos usuários. Palavras-chave: Profissionais de saúde; Ervas medicinais; Atenção básica.
\end{abstract}

\begin{abstract}
Ethnopharmacology deals with popular knowledge concerning traditional systems of medicine; it consists of information acquired throughout life by the population using medicinal flora as pharmacological study. The aim is to analyze the scientific evidence of health professionals' knowledge of the use of ethnopharmacology as a complementary therapy in primary care. An integrative literature review was conducted using the online search platforms Us National Library of Medicine (PUBMED) and Virtual Health Library (VHL) in the databases LILACS, MEDLINE and BDENF. To construct the research question of the integrative review, the Pico strategy was used, essential for increasing the specificity and conceptual clarity of the situation to be studied, providing results of greater quality and uniqueness. Articles indexed in national and international journals were selected, provided in full, and studies found in the last 5 years in Portuguese, English and Spanish. The results suggest that although phytotherapy is a beneficial practice for many, there are still factors that act as barriers to the consolidation of this therapy within health services. Therefore, it becomes extremely important to mobilize strategies that can pave the way for the effective implementation of ethnopharmacological practices as an integral part of the users' health management.
\end{abstract}

Keywords: Health professionals; Medicinal herbs; Primary health care.

\section{Resumen}

La etnofarmacología se ocupa del conocimiento popular relacionado con los sistemas tradicionales de medicina, consiste en la información adquirida a lo largo de la vida por la población que utiliza la flora medicinal con el estudio farmacológico. El objetivo es analizar las evidencias científicas sobre el conocimiento de los profesionales de la salud acerca del uso de la etnofarmacología como terapia complementaria en la atención básica. Para ello, se realizó una revisión bibliográfica integradora utilizando las plataformas de investigación online Us national Library of Medicine 
(PUBMED) y Virtual Health Library (BVS) en las bases de datos LILACS, MEDLINE y BDENF. Para construir la pregunta de investigación de la revisión integradora, se utilizó la Estrategia del Pico, esencial para aumentar la especificidad y la claridad conceptual de la situación a estudiar, proporcionando resultados de mayor calidad y singularidad. Se seleccionaron artículos indexados en revistas nacionales e internacionales, proporcionados en su totalidad, y estudios encontrados en los últimos 5 años en portugués, inglés y español. Los resultados sugieren que, aunque la fitoterapia es una práctica beneficiosa para muchos, todavía hay factores que actúan como barreras para la consolidación de esta terapia dentro de los servicios sanitarios. Por lo tanto, se vuelve extremadamente importante la movilización de estrategias que puedan abrir caminos para la implementación efectiva de las prácticas etnofarmacológicas como parte integral del manejo de la salud de los usuarios.

Palabras clave: Profesionales de la salud; Hierbas medicinales; Atención primaria de salud.

\section{Introdução}

A utilização das plantas para fins terapêuticos é tão antiga quanto as civilizações, pode-se destacar como tradições medicinais as culturas indianas e a chinesa que são bastante aplicadas para fins curativos. As plantas medicinais são deveras utilizadas na busca de novos medicamentos como também fitoterápicos, e conforme a ANVISA há diferença entre os termos plantas medicinais e medicamentos fitoterápicos, onde se considera o primeiro termo como plantas utilizadas para tratar, prevenir ou curar alguma doença e o segundo termo diz respeito a toda substância de origem vegetal e que sua ação, eficácia e efeitos foram cientificamente comprovadas (Santos \& Trindade, 2017). A medicina passou por profundas modificações temporais e adquiriu características marcantes. No entanto, as tradições de bases naturais ainda vigoram e encontram aceitação e prática por diversas comunidades (Lima et al., 2017).

A etnofarmacologia trata do conhecimento popular referente aos tradicionais sistemas da medicina, ela consiste em informações adquiridas ao longo da vida pela população usuária da flora medicinal com o estudo farmacológico. A ordenação etnofarmacológica permite que hipóteses sejam formuladas para saber se determinada substância ativa presente, está relacionada ao melhoramento de ações terapêuticas expostas. Entretanto, no Brasil a inclusão de plantas como medicamento na cultura ainda é pouco difundida, apesar de que em regiões como a Amazônia, Cerrado e Mata Atlântica elas serem muito utilizadas (Sampaio et al., 2012).

Em outra face o uso de medicamentos industrializados é muito divulgado pela indústria farmacêutica, porém, grande parte da população brasileira busca outras possibilidades para aliviar alguns sintomas ou para a cura de algumas enfermidades, pois, esses meios possuem menos efeitos colaterais do que os fármacos tradicionais. No entanto, mesmo sendo plantas naturais, julga-se que por sua composição química conter diversos princípios ativos, eles podem provocar várias avarias ao organismo humano se usados de maneira indevida (Silva Júnior et al., 2017).

O tema se torna tão relevante que a OMS tem expressado seu posicionamento a favor da valorização dessas práticas como forma de fortalecer a cobertura da atenção primaria à saúde e diferir da terapêutica vigente e hegemônica, atualmente, nos cuidados a saúde, que se caracteriza como essencialmente medicamentosa (Freire et al., 2014). As vantagens do manejo da conduta etnofarmacológica beneficia nos dois sentidos: a comunidade cientifica, por meio do fornecimento, pela população, de informações pertinentes para o desenvolvimento de estudos farmacológicos; e aos usuários, pela redução dos custos com medicamentos (Sampaio et al., 2012).

A nível brasileiro, também foi desenvolvido métodos para a articulação e implementação da etnofarmacologia no âmbito da saúde. A exemplo foi o Programa Nacional de Plantas Medicinais e Fitoterápicos, que tem como algumas das diretrizes básicas a ampliação das opções terapêuticas e melhoria da atenção à saúde aos usuários do SUS. Conforme estimulado pela OMS, aprovou-se também, em 2006, a Política Nacional de Práticas Integrativas e Complementares no SUS, que entre outros, dá suporte ao uso de plantas medicinais e fitoterápicos, e visa promover a racionalização das condutas de saúde, encorajando

alternativas transformadoras e popularmente contributivas ao desenvolvimento sustentável das comunidades (Ministério da 
Saúde, 2015).

Como atividade essencial do enfermeiro, o ato de cuidar também abrange o acolhimento, a valorização das singularidades dos pacientes, a promoção da autonomia como forma da geração de vínculo e confiança, a contribuição cientifica para o melhoramento das técnicas já utilizadas. No entanto, é perceptível a baixa adesão dessa modalidade pelos profissionais da saúde. Os fatores que limitam o desenvolvimento se traduzem pela falta de conhecimento cientifico, evidenciado pela precariedade do ensino acadêmico ou falta de interesse pela área; desconfiança quanto a qualidade e eficácia desse método; tanto pela escassez no desenvolvimento de estudos que evidenciem a relevância e aplicabilidade da temática no âmbito da saúde quanto da potencialidade do Brasil produzir essa classe terapêutica, apesar de uma flora brasileira riquíssima. Todos esses fatores corroboram para uma prática ainda muito tímida e limitada (Sampaio et al., 2012).

\section{Metodologia}

$\mathrm{O}$ estudo tratou-se de uma revisão integrativa de caráter quantitativo, investigou conhecer o conhecimento dos profissionais de saúde acerca do uso das plantas medicinais e fitoterápicos na atenção básica de saúde, outrossim, a pesquisa científica tem a finalidade de elaborar conhecimento ou solução para o tema em debate, culminando na transformação e compreensão da realidade, que está integrada a contexto sócio-históricos. As etapas foram: construção da questão de pesquisa, amostragem, classificação dos estudos, avaliação dos estudos incluídos, interpretação e síntese dos resultados.

O levantamento dos estudos foi realizado em agosto a setembro de 2021, por meio do acesso virtual às bases de dados: Literatura Latino Americana e do Caribe em Ciências da Saúde (LILACS); Bases de Dados bibliográficos especializados na área de Enfermagem (BDENF) por meio da consulta à Biblioteca Virtual em Saúde (BVS); Medical Literature Analysis and Retrieval System Online (MEDLINE), acessada por meio do portal PUBMED. A busca dos artigos foi realizada nos Descritores em Ciências da Saúde (DeCS), e cruzadas entre si por meio do operador booleano "AND”. As palavras chaves foram: terapias de ervas; assistência de enfermagem; Terapias Alternativas; Ervas Medicinais.

Por isso, "P" refere-se ao problema do estudo (conhecimento dos profissionais de saúde), "I" refere-se a variável de interesse (uso da etnofarmacologia); "C" ao resultado/contexto: (uso da etnofarmacologia na atenção primária à saúde).

Quadro 1: Estratégia para busca e seleção de estudos. Teresina-PI, 2021.

\begin{tabular}{|l|l|l|}
\hline $\begin{array}{l}\text { P (população/problema): } \\
\text { conhecimento dos profissionais } \\
\text { de saúde }\end{array}$ & $\begin{array}{l}\text { I (variável de interesse): uso da } \\
\text { etnofarmacologia }\end{array}$ & $\begin{array}{l}\text { Co (resultado/contexto); uso da } \\
\text { etnofarmacologia na atenção primária á } \\
\text { saúde }\end{array}$ \\
\hline $\begin{array}{l}\text { Decs: } \\
\text { Pessoal de Saúde }\end{array}$ & $\begin{array}{l}\text { Decs: } \\
\text { Plantas medicinais } \\
\text { Fitoterapia } \\
\text { Terapias complementares } \\
\text { Atenção Primária à Saúde }\end{array}$ \\
\hline $\begin{array}{l}\text { Palavras-chaves: } \\
\text { Profissionais de Saúde; } \\
\text { Trabalhadores de Saúde }\end{array}$ & $\begin{array}{l}\text { Palavras-chaves: } \\
\text { Terapias Alternativas; Ervas Medicinais. }\end{array}$ & $\begin{array}{l}\text { Palavras-chaves: } \\
\text { Atenção Básica; Primeiro Nivel de } \\
\text { Assistência }\end{array}$ \\
\hline $\begin{array}{l}\text { Mesh: } \\
\text { Health Personnel }\end{array}$ & $\begin{array}{l}\text { Mesh: } \\
\text { Plants medicinal } \\
\text { Primary Health Care } \\
\text { Chytotherapy }\end{array}$ & \\
\hline
\end{tabular}

Fonte: Maria Clara Barradas Leão e Yanka Delmondes Campelo. Teresina-PI, 2021.

Desse modo, a delimitação da questão de pesquisa foi: "Qual o nível de conhecimento dos profissionais de saúde sobre o uso da etnofarmacologia na atenção primária à saúde?". Como critérios de inclusão foram selecionados: estudos primários de 
abordagem qualitativa e quantitativa; estudos que reportem aspectos do papel do profissional de saúde com relação a temática; estudos disponíveis nos idiomas português e inglês com recorte temporal dos últimos cinco anos. Como critérios de exclusão, foram excluídos tipos de estudos, como: cartas, editoriais, resumos, teses e dissertações também serão excluídas. Combinando todas as bases de dados foram encontrados 12 artigos como mostra a tabela.

Figura 1: Fluxograma do processo de busca dos artigos, 2021.

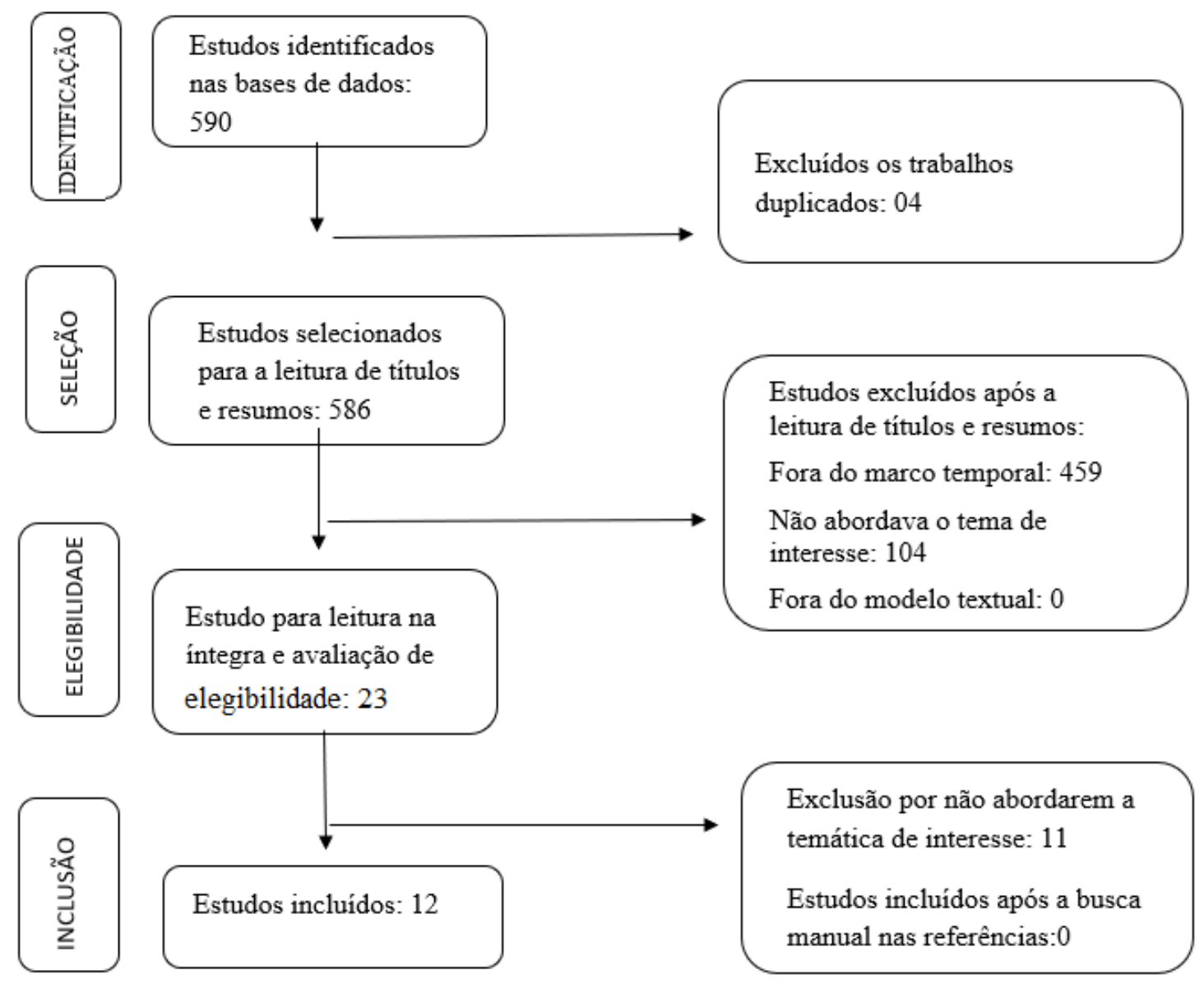

Fonte: PRISMA Flow Diagram.

A construção do fluxograma no processo de busca de artigos foi importante pois demonstra as etapas que foram percorridas para chegar até até os artigos que foram selecionados para dar suporte ao artigo.

Após a leitura dos títulos dos artigos, notou-se que alguns deles se repetiram nas diferentes bases e outros não atestaram os critérios de estudo. Ao todo, foram elegidos 586 artigos para leitura do resumo e excluídos os que não atendiam ao estudo e para a leitura na íntegra foram selecionados 23 artigos e excluídos os que não abordavam a temática de interesse. Após a leitura na íntegra, foram selecionados 12 artigos para análise e categorização.

\section{Resultados e Discussão}

A partir da leitura e análise do material achado conforme os critérios de elegibilidade determinados no tópico de metodologia, foram reunidas informações pertinentes a respeito dos artigos encontrados, entre os quais: ano de publicação, abordagem metodológica, e periódico de publicação, como demonstra a Tabela 1. 
Tabela 1: Categorização de artigos conforme Ano, Abordagem Metodológica e Periódico de Publicação. Teresina-PI, 2021.

\begin{tabular}{lcc}
\hline & VARIÁVEIS & \\
\hline ANO DE PUBLICAÇÃO & $\mathbf{N}^{\mathbf{0}}$ & $\%$ \\
2016 & 3 & 30 \\
2017 & 5 & 50 \\
2018 & 5 & 20 \\
2019 & 2 & 10 \\
2021 & 1 & 10
\end{tabular}

\section{ABORDAGEM METODOLÓGICA}

Revisão de Literatura

\section{PERIÓDICO DE PUBLICAÇÃO}

\begin{tabular}{lll} 
Brazilian Journal of Biology & 1 & 10 \\
Complementary and Alternative Medicine & 2 & 20 \\
Complementary Therapies in Medicine & 1 & 10 \\
International Journal of Nursing Studies & 1 & 10 \\
Patient Education and Couseling & 1 & 10 \\
Primary Health Care Research \& Development & 1 & 10 \\
Qualitative Health Research & 1 & 10 \\
Revista APS & 1 & 10 \\
Revista Ciência e Saúde Coletiva & 1 & 10 \\
Revista Gaúcha de Enfermagem & 1 & 10 \\
Vaccine & 1 & 10 \\
\hline
\end{tabular}

Fonte: Autores.

Como demonstra a tabela anterior, observa-se grande distribuição entre os anos de publicação, contudo, o ano de 2017 apresenta maior periodicidade de publicação de artigos sobre a temática, totalizando 5 publicações, o que representa $50 \%$ do total de artigos estudado, com relação a abordagem metodológica 100\% foram pesquisas de Revisão de Literatura. Nas publicações, vale ressaltar a variedade de periódicos, sendo $20 \%$ dos artigos publicados na revista Complementary and. Alternative Medicine, e cada um dos outros periódicos expostos na tabela 3 com $10 \%$ de publicação.

Analisando-se a amostra de artigos incorporados nesta revisão, os estudos foram, em sua maioria, desenvolvidos no Brasil $(n=4,33,33 \%)$ e na Austrália $(n=4,33,33 \%)$, seguidos da Alemanha $(n=1,8,33 \%)$, África do Sul $(n=1,8,33 \%)$, China $(\mathrm{n}=1,8,33 \%)$ e Reino Unido $(\mathrm{n}=1,8,33 \%)$. Quanto ao idioma, os estudos foram publicados em Inglês $(\mathrm{n}=9$, 75\%) e Português $(n=3,25 \%)$.

Devido a atualidade do tema abordado, todas as publicações são datadas dos anos de $2021(\mathrm{n}=1,8,33 \%), 2019(\mathrm{n}=1$, 8,33\%), $2018(\mathrm{n}=2,16,66 \%), 2017(\mathrm{n}=5,41,66 \%) 2016(\mathrm{n}=3,25 \%)$.

Houve a presença de diferentes periódicos, dentre eles: Int. J Nurs Stud ( $\mathrm{n}=1,8,33 \%)$, Rev Gaúcha Enferm ( $\mathrm{n}=1$, 8,33\%), Qual Health Res ( $\mathrm{n}=1,8,33 \%)$, Complement Ther Med ( $\mathrm{n}=1,8,33 \%)$, Prim Health Care Res $(\mathrm{n}=1$, 8,33\%), Patient Educ Couns $(n=1,8,33 \%)$, Complement Altern Med $(n=2,16,66 \%)$, Vaccine $(n=1,8,33 \%)$, Ciênc. Saúde Colet $(n=1$, 8,33\%), Braz. J. Biol $(\mathrm{n}=1,8,33 \%)$ e Rev. APS $(\mathrm{N}=1,8,33 \%)$. As características dos estudos incluídos encontram-se detalhadas no quadro.

No que concerne ao delineamento metodológico empregado nos registros incluídos neste estudo, os desenhos concentram-se em: estudo qualitativo $(n=3,25 \%)$, estudo exploratório $(n=2,16,66 \%)$, revisão sistemática e metassíntese $(n=$ $1,8,33 \%)$, estudo quantitativo $(n=1,8,33 \%)$, estudo transversal $(n=1,8,33 \%)$, revisão sistemática $(n=1,8,33 \%)$, estudo 
quantitativo de corte transversal $(\mathrm{n}=1,8,33 \%)$, estudo observacional quantitativo $(\mathrm{n}=1,8,33 \%)$, estudo exploratório qualitativo $(n=1,8,33 \%)$.

Quadro 2: Registros incluídos pela revisão de escopo, segundo Título, Periódico, País de origem e Ano. Teresina-PI, 2021.

\begin{tabular}{|c|c|c|c|}
\hline Título do artigo & $\begin{array}{l}\text { Título abreviado } \\
\text { do periódico }\end{array}$ & País & Ano \\
\hline $\begin{array}{l}\text { Nurses' attitudes towards complementary therapies: A systematic review and meta- } \\
\text { synthesis }\end{array}$ & Int J Nurs Stud. & Austrália & 2017 \\
\hline $\begin{array}{l}\text { Práticas integrativas e complementares como recurso de saúde mental na Atenção } \\
\text { Básica }\end{array}$ & $\begin{array}{l}\text { Rev Gaúcha } \\
\text { Enferm }\end{array}$ & Brasil & 2017 \\
\hline $\begin{array}{l}\text { Collaboration Between Biomedical and Complementary and Alternative Care } \\
\text { Providers: Barriers and Pathways }\end{array}$ & Qual Health Res & $\begin{array}{l}\text { África do } \\
\text { sul }\end{array}$ & 2017 \\
\hline $\begin{array}{l}\text { Delivery of patient-centered care in complementary medicine: Insights and } \\
\text { evidence from the Chinese medical practitioners and patients in primary care } \\
\text { consultations in Hong Kong }\end{array}$ & $\begin{array}{l}\text { Complement Ther } \\
\text { Med. }\end{array}$ & China & 2019 \\
\hline $\begin{array}{l}\text { Primary care practitioner perceptions and attitudes of complementary medicine: a } \\
\text { content analysis of free-text responses from a survey of non-metropolitan } \\
\text { Australian general practitioners }\end{array}$ & $\begin{array}{l}\text { Prim Health Care } \\
\text { Res }\end{array}$ & Austrália & 2018 \\
\hline $\begin{array}{l}\text { Complementary and alternative medicine (CAM) as part of primary health care in } \\
\text { Germany-comparison of patients consulting general practitioners and CAM } \\
\text { practitioners: a cross-sectional study }\end{array}$ & Altern Med & Alemanha & 2016 \\
\hline $\begin{array}{l}\text { Patient perceptions of clinical care in complementary medicine: A systematic } \\
\text { review of the consultation experience }\end{array}$ & $\begin{array}{l}\text { Patient } \\
\text { Couns. }\end{array}$ & Austrália & 2016 \\
\hline $\begin{array}{l}\text { 'Trying to put a square peg into a round hole': a qualitative study of healthcare } \\
\text { professionals' views of integrating complementary medicine into primary care for } \\
\text { musculoskeletal and mental health comorbidity }\end{array}$ & $\begin{array}{l}\text { Complement } \\
\text { Altern Med }\end{array}$ & $\begin{array}{l}\text { Reino } \\
\text { Unido }\end{array}$ & 2018 \\
\hline $\begin{array}{l}\text { The associations between medical, allied and complementary medicine practitioner } \\
\text { visits and childhood vaccine uptake }\end{array}$ & Vaccine & Australia & 2017 \\
\hline $\begin{array}{l}\text { Plantas medicinais e fitoterápicos na Atenção Primária em Saúde: } \\
\text { percepção dos profissionais }\end{array}$ & Ciênc. Saúde Colet & Brasil & 2016 \\
\hline Situational diagnosis of professionals of family health units on phytotherapy & Braz. J. Biol & Brasil & 2021 \\
\hline Plantas medicinais e sua aplicabilidade na atenção primária à saúde & Rev. APS & Brasil & 2017 \\
\hline
\end{tabular}

Fonte: Autores.

Baseado nos resultados obtidos a partir do cruzamento entre os descritores mencionados no quadro 1 , um total de 12 artigos foram selecionados para fundamentar este estudo, sendo dividido conforme demonstra o quadro 3, sendo classificados por Título, Autores, Ano de publicação e principais resultados. 
Quadro 3: Categorização de artigos por Título, Autoria, Ano de publicação e principais resultados. Teresina-PI, 2021.

\begin{tabular}{|c|c|c|c|}
\hline TÍTULO & AUTORES & ANO & PRINCIPAIS RESULTADOS \\
\hline $\begin{array}{l}\text { Patient perceptions of clinical care in } \\
\text { complementary medicine: A systematic } \\
\text { review of the medicine: A systematic } \\
\text { review of the consultation experience }\end{array}$ & $\begin{array}{l}\text { Foley \& } \\
\text { Steel }\end{array}$ & 2016 & $\begin{array}{l}\text { This review aims to describe the prevalence of empathy, empowerment } \\
\text { and patient-centred clinical care experienced by patients in } \\
\text { complementary medicine (CM) consultations. Methods: A systematic } \\
\text { review was undertaken of original research exploring patient perceptions } \\
\text { of CM clinical care. Ten databases were searched: Alt HealthWatch, } \\
\text { AMED, CINAHL Plus, MEDLINE Complete, Cochrane Library, } \\
\text { PubMed, Proquest Medical Collection, PsycInfo, Social Sciences } \\
\text { Citation Index and Psychology Collection. Studies were included which } \\
\text { reported patient perceptions of consultation with CM practitioners and } \\
\text { were excluded where experimental methods controlled the nature of } \\
\text { consultation processes. }\end{array}$ \\
\hline $\begin{array}{l}\text { Plantas medicinais e fitoterápicos na } \\
\text { Atenção Primária em Saúde: percepção } \\
\text { dos profissionais }\end{array}$ & Matos et al. & 2016 & $\begin{array}{l}\text { identificar conhecimentos e práticas em relação à prescrição e/ou } \\
\text { sugestão de uso de plantas medicinais e fitoterápicos pelos profissionais } \\
\text { de saúde, a fim de destacar pontos positivos, dificuldades, necessidades, } \\
\text { sinalizando estratégias que contribuam para a implementação desta } \\
\text { terapia no município }\end{array}$ \\
\hline $\begin{array}{l}\text { Complementary and alternative } \\
\text { medicine (CAM) as part of primary } \\
\text { health care in Germany-comparison of } \\
\text { patients consulting general } \\
\text { practitioners and CAM practitioners: a } \\
\text { cross-sectional study }\end{array}$ & Krug et al. & 2016 & $\begin{array}{l}\text { Considering the steadily growing number of unregulated HPs, the aim of } \\
\text { the study was to assess characteristics of patients consulting HPs in } \\
\text { comparison to patients consulting GPs }\end{array}$ \\
\hline $\begin{array}{l}\text { Nurses' attitudes } \quad \text { towards } \\
\text { complementary therapies: A systematic } \\
\text { review and meta-synthesis }\end{array}$ & Hall et al. & 2017 & $\begin{array}{l}\text { The aim of this meta-synthesis is to review, critically, appraise and } \\
\text { synthesize the existing qualitative research to develop a new, more } \\
\text { substantial interpretation of nurses' attitudes regarding the, use of } \\
\text { complementary therapies by patients }\end{array}$ \\
\hline $\begin{array}{l}\text { Collaboration Between Biomedical and } \\
\text { Complementary and Alternative Care } \\
\text { Providers: Barriers and Pathways }\end{array}$ & Watt et al. & 2017 & $\begin{array}{l}\text { This study provides a novel cross-national insight into the perspectives } \\
\text { of collaboration from four stakeholder groups }\end{array}$ \\
\hline $\begin{array}{l}\text { Práticas integrativas e complementares } \\
\text { como recurso de saúde mental na } \\
\text { Atenção Básica }\end{array}$ & $\begin{array}{l}\text { Carvalho \& } \\
\text { Nóbrega }\end{array}$ & 2017 & $\begin{array}{l}\text { O conhecimento dos profissionais precisa ser aprofundado. Ainda assim, } \\
\text { os mesmos consideram as PIC como um recurso em Saúde Mental na } \\
\text { Atenção Básica. }\end{array}$ \\
\hline $\begin{array}{l}\text { Plantas medicinais e sua aplicabilidade } \\
\text { na atenção primária à saúde }\end{array}$ & Ceolin et al. & 2017 & $\begin{array}{l}\text { Fica evidente a necessidade de repensar a formação e qualificação } \\
\text { dos profissionais da saúde para que possam atender } \\
\text { à demanda da população sobre o cuidado com plantas }\end{array}$ \\
\hline $\begin{array}{l}\text { The associations between medical, } \\
\text { allied and complementary medicine } \\
\text { practitioner visits and childhood } \\
\text { vaccine uptake }\end{array}$ & $\begin{array}{l}\text { Frawley, } \\
\text { Foley \& } \\
\text { McIntyre }\end{array}$ & 2017 & $\begin{array}{l}\text { Os pais que não vacinaram seus filhos parecem confiar em fontes não } \\
\text { convencionais de informação, como os praticantes de MC. Mais } \\
\text { pesquisas são necessárias para determinar como gerenciar os desafios e } \\
\text { oportunidades dos médicos de CM como fonte de informações sobre } \\
\text { vacinas. }\end{array}$ \\
\hline $\begin{array}{l}\text { Primary care practitioner perceptions } \\
\text { and attitudes of complementary } \\
\text { medicine: a content analysis of free- } \\
\text { text responses from a survey of non- } \\
\text { metropolitan Australian general } \\
\text { practitioners }\end{array}$ & $\begin{array}{l}\text { Wardle, } \\
\text { Sibbritt, } \\
\text { David \& } \\
\text { Adams }\end{array}$ & 2018 & $\begin{array}{l}\text { This study examines GP perceptions, attitudes and knowledge of } \\
\text { complementary medicine (CM), and to understand contextual factors } \\
\text { that influence these perceptions, attitudes and knowledge }\end{array}$ \\
\hline $\begin{array}{l}\text { 'Trying to put a square peg into a round } \\
\text { hole': a qualitative study of healthcare } \\
\text { professionals' views of integrating } \\
\text { complementary medicine into primary } \\
\text { care for musculoskeletal and mental } \\
\text { health comorbidity }\end{array}$ & Sharp et al. & 2018 & $\begin{array}{l}\text { CAM tem o potencial de ajudar o NHS no tratamento da carga da } \\
\text { comorbidade MSK-MH. Dados os desafios da integração, a } \\
\text { incorporação seletiva usando o encaminhamento tradicional da atenção } \\
\text { primária para o CAM pode ser o modelo mais viável }\end{array}$ \\
\hline $\begin{array}{l}\text { Delivery of patient-centered care in } \\
\text { complementary medicine: Insights and } \\
\text { evidence from the Chinese medical } \\
\text { practitioners and patients in primary } \\
\text { care consultations in Hong Kong }\end{array}$ & $\begin{array}{l}\text { Pun, Chor \& } \\
\text { Zhong }\end{array}$ & 2019 & $\begin{array}{l}\text { As estratégias específicas identificadas no estudo atual podem melhorar } \\
\text { a comunicação dos profissionais da MTC com os pacientes, criando um } \\
\text { ambiente que certamente otimizará a segurança para pacientes e } \\
\text { médicos. }\end{array}$ \\
\hline $\begin{array}{l}\text { Situational diagnosis of professionals } \\
\text { of family health units on phytotherapy. }\end{array}$ & Bezerra & 2021 & $\begin{array}{l}\text { Os profissionais de saúde têm conhecimento limitado sobre o assunto e } \\
\text { demonstraram interesse em participar de cursos/treinamentos sobre } \\
\text { fitoterapia. }\end{array}$ \\
\hline
\end{tabular}

Fonte: Autores. 
Os artigos mencionados no quadro anterior foram organizados de forma crescente por ano de publicação para uma melhor visualização e classificação, os principais resultados conseguidos consistem em um resumo a partir da leitura dos estudos e trata dos principais achados em cada publicação.

As terapias complementares são um conjunto de práticas que abrangem duas categorias: produtos naturais e práticas corporais. Apesar da evolução das tecnologias convencionais de saúde, as terapias alternativas estão em popularidade. No entanto, é necessário ter cuidado, pois a suposição de que tudo que é "natural" trará benefícios pode colocar em risco a integridade do indivíduo. Nesse contexto se faz extremamente importante o conhecimento prévio acerca do tema e suas peculiaridades dentre os profissionais de saúde (Hall et al., 2017). Em consonância a isso Krug et al. (2016), confirma que a medicina complementar não é livre de riscos, pois há comprovadamente interação entre medicamentos tradicionais e substâncias naturais. A ausência de relato dos pacientes sobre o uso dessas substâncias, por receito de julgamentos, contribui para o manejo inadequado da terapêutica. Baseado nos seus estudos foi possível concluir que pacientes em uso de terapias alternativas melhoraram padrão de exercício físico, diminuição do tabagismo e melhora na autoavaliação.

De acordo com Carvalho e Nobrega (2017), as práticas complementares incluem sistemas e recursos que valorizam a escuta e o abrigo desenvolvendo laços terapêuticos e integrando o ser humano no seu contexto. A disponibilização dessas alternativas na Atenção Primária à Saúde além de um método terapêutico, funciona também como promoção da saúde e oferta de cuidados holísticos. Segundo Sharp et al. (2018), a medicina integrativa visa transformar a saúde, incorporando uma abordagem holística e proporcionando tratamento respeitando qualquer tradição. Além de acreditar que a atenção primária é o âmbito mais favorável para o desenvolvimento da integração de abordagens convencionais com abordagens complementares, pois têm uma perspectiva em comum: a ênfase no autocuidado.

De acordo com Ceolin et al. (2017), a atenção a saúde tem uma forte tendência do uso de tecnologias diagnóstica terapêutica, e paralelamente a isso um tímido recrudescimento de terapias complementares. O cenário encontrado nos ambientes de saúde tem forte influência no desenvolvimento dessas práticas, pois a implementação dessa modalidade requer uma abertura dos profissionais que estão dispostos a desenvolvê-las, o reposicionamento dos usuários para o centro do modelo do cuidado e o resgate de práticas milenares através do diálogo entre conhecimento técnico e popular. E concluiu-se, com seu estudo, que quando os profissionais são capacitados de forma eficiente, são capazes de responder a orientação correta do manejo e uso das substâncias fitoterápicas.

Já na visão de Sharp et al. (2018), entre os profissionais de saúde, as percepções são variadas e encontram como barreiras o ceticismo profissional, não sendo notado fatores pessoais envolvidos. Dentre o papel das terapias complementares, no seu estudo, concluiu-se que essas terapias tem papel importante na assistência à saúde, embora faltasse prática e entusiasmo pelos profissionais.

Ainda, os problemas que podem ser identificados podem ser: medicina convencional; culturas corporativas; falta de comunicação e colaboração entre as equipes. Na visão de Carvalho e Nobrega (2017), a barreira encontrada para o desenvolvimento dessas práticas é a gestão pública, traduzida em baixos recursos humanos, financiamentos, além da lógica biomédica. Outro fator importante destacado é o papel da Atenção Primária à Saúde no contexto da multiplicação dessas práticas, pois desde a institucionalização do modelo de da Estratégia de Saúde da Família, criada pelo SUS, que tem como objetivos e diretrizes a reorientação do modelo de assistência para uma perspectiva psicossocial, ressaltando a necessidade de um cuidado integral. Foi concluído segundo o autor que Quanto ao conhecimento dos profissionais sobre o termo "Práticas Complementares", a resposta encontrada foi afirmativa em $76,8 \%$, enquanto o conhecimento sobre PCs era encontrado em $73,9 \%$ dos casos.

Ainda sobre as principais barreiras para a colaboração das práticas populares em conjunto com as práticas biomédicas, Watt et al. (2017) revela: desconfiança, restrições geográficas e número de profissionais capacitados. Segundo ele os países em 
desenvolvimento incorporam essa modalidade terapêutica no sistema formal de saúde desde a década de 70. Wardle, Sibbritt e Adams (2018) concordam em afirmar que o uso dessas práticas tem crescido no mundo todo, encontrando na Austrália número quase semelhante entre a procura por consultas convencionais e as consultas de terapias alternativas.

Conforme Bezerra (2016), a capacitação dos profissionais acerca do conhecimento das propriedades, modo de preparo, potencial tóxico, interações medicamentosas e alimentares são indispensáveis para que haja um manejo adequado do uso da fitoterapia pelos usuários de saúde. Em confronto com isso, revela-se em estudo que os profissionais em sua maioria desconhecem os efeitos de interações dessas substâncias. Bezerra (2016) e Sharp et al. (2018) são consoantes em mostrar em seus estudos que há uma boa aceitação da incorporação das práticas etnofarmacológicas nos serviços de saúde, evidenciado por uma porcentagem significativa.

Em conformidade com Foley e Steel (2017), é perceptível o uso das medidas complementares pela população, principalmente entre aquelas com condições crônicas de saúde, além de uma maior eficácia e abrangência da combinação de terapias convencionais às alternais nos cuidados primários à saúde. Não se deve subestimar a importância da consulta convencional, pois ela é parte fundamental para os diagnósticos de saúde e orienta as demais terapêuticas a serem instituídas. Foram encontrados nos resultados aos temas abrangentes de empatia, empoderamento e concentração no paciente cuidados foram refletidos em estudos qualitativos através do uso de linguagem, ênfase e contextualização similares. Estas categorias serviram para caracterizar a experiência do paciente acerca do tema

A enfermagem constitui uma das maiores forças de trabalho no âmbito da saúde, além de desempenhar papéis centrados no bem estar do cidadão. Além disso, os enfermeiros são mais acessíveis e capazes de se envolver na valorização dessas terapias quando referidas pelos pacientes, influenciando dessa forma, o manejo adequado das terapias etnofarmacológicas. Enquanto os resultados de Foley e Steel (2017) foram centrados na percepção dos clientes, esse estudo mostrou cinco temas analíticos relacionados com as atitudes dos enfermeiros em relação as terapias complementares surgiram a partir dos dados: os pontos fortes e fraquezas da medicina convencional; terapias complementares como forma de melhorar a prática da enfermagem; capacitação e centralização do paciente; barreiras culturais e capacitadores para integração (Hall et al., 2017).

\section{Conclusão}

A fitoterapia pode ser usada como ferramenta alternativa e complementar na prestação de cuidados, valorizando a cultura e conhecimento dos usuários de saúde. O uso de plantas medicinais e a fitoterapia ainda são pouco difundidos nos sistemas de saúde. Alguns dos fatores relacionados são: a falta de abordagem do assunto nas graduações; pois essas seguem o modelo essencialmente biomédico, fazendo com que a atuação dos profissionais siga esse modelo, a não aceitação de alguns profissionais de saúde em utilizar as técnicas etnofarmacológicas; por acreditarem na supremacia da medicina tradicional, e a falta de estímulos pelos gestores em qualificar e preparar esses profissionais para integrar na sua rotina de serviços tais técnicas; além da desconfiança da qualidade e eficácia do método.

Esse estudo pode ser usado como sugestão para a produção de demais estudos para agregar conhecimento sobre a importância do uso de terapêuticas alternativas para a prestação de um cuidado mais amplo, integrando o conhecimento científico e os valores e crenças populares que irão contribuir para a autonomia do paciente, tornando-o personagem principal na promoção e manutenção de sua saúde. Além de expor a deficiência de conhecimento, que é indicada pelo baixo número de profissionais que aceitam, conhecem ou utilizam essas técnicas como ferramenta na atenção à saúde. 


\section{Referências}

Aguiar, J., Kanan, L. A., \& Masiero, A. V. (2020). Práticas Integrativas e Complementares na atenção básica em saúde: um estudo bibliométrico da produção brasileira. Saúde em Debate. 43(123), 1205-1218.

Anvisa. (2016). Memento Fitoterápico da Farmacopeia Brasileira. http://www.farmacia.pe.gov.br/sites/farmacia.saude.pe.gov.br/files/memen to_fitoterapico.pdf

Bezerra, A. S. C. E. (2016). Diagnóstico situacional dos profissionais das unidades de saúde da família sobre a fitoterapia. Dissertação de mestrado, Centro Universitário CESMAC, Maceió, Alagoas, Brasil.

Boas, R. M. V. (2020). Conhecimento popular e utilização de chás de plantas medicinais: uma revisão narrativa da literatura. Monografia, Faculdade Maria Milza, Governador Mangabeira, Bahia, Brasil.

Carvalho, J. L. S., \& Nóbrega, M. P. S. S. (2018). Complementary therapies as resources for mental health in Primary Health Care. Revista Gaucha de Enfermagem. 38(4), 1-9.

Ceolin, S., Ceolin, T., Casarin, S. T., Severo, V. O., Ribeiro, M. V., \& Lopes, A. C. P. (2017). Plantas medicinais e sua aplicabilidade na atenção primária à saúde. Revista de APS. 20(1), 81-88.

Estrela, C. (2018). Metodologia científica: ciência, ensino, pesquisa. Artes Médicas.

Ferreira, E. T., Santos, E. S., Monteiro, J. S., Gomes, M. S. M., Menezes, R. A. O., \& Souza, M. J. C. (2019). A utilização de plantas medicinais e fitoterápicos: uma revisão integrativa sobre a atuação do enfermeiro. Brazilian Journal of Health Review. 2(3), $1511-1523$.

Flores, G. E., Oliveira, D. L. L., \& Zocche, D. A. A. (2016). Educação permanente no contexto hospitalar: a experiência que ressignifica o cuidado em enfermagem. Trabalho, Educação e Saúde. 14(2), 487-504.

Foley, H., \& Steel, A. (2017). Patient perceptions of clinical care in complementary medicine: A systematic review of the consultation experience. Patient education and counseling. 100(2), 212-223.

Frawley, J. E., Foley, H., \& McIntyre, E. (2018). The associations between medical, allied and complementary medicine practitioner visits and childhood vaccine uptake. Vaccine. 36(6), 866-872.

Hall, H. Leach, M., Brosnan, G., \& Collins M. (2017). Nurses' attitudes towa0rds complementary therapies: A systematic review and metasynthesis. International Journal of Nursing Studies. 69, 47-56.

Krug, K., Kraus, K. I., Herrmann, K., \& Joos, S. (2016). Complementary and alternative medicine (CAM) as part of primary health care in Germanycomparison of patients consulting general practitioners and CAM practitioners: a cross-sectional study. BMC complementary and alternative medicine. 16(1), $1-8$.

Lima, R. F. S., Turrini, R. N. T., Silva, L. R., Melo, L. D. S., \& Augusto, S. I. (2017). Práticas populares de cura e o uso de plantas medicinais por mães ribeirinhas no cuidado infantil. Rev. Pesqui. 9(4), 1154-1163.

Lopes, A. C. P., Ceolin, T., Ceolin, S., \& Lopes, C. V. (2018). As contribuições da disciplina "Terapias complementares com ênfase em plantas medicinais" na prática profissional dos enfermeiros. Rev. pesqui. cuid. fundam. 10(3), 619-625.

Mattos, G., Camargo, A., Sousa, C. A., \& Zeni, A. L. B. (2018). Plantas medicinais e fitoterápicos na Atenção Primária em Saúde: percepção dos profissionais. Ciência \& Saúde Coletiva. 23(11), 3735-3744.

Mattos, G., Camargo, A., Sousa, C. A., Zeni, A. L. B. Plantas medicinais e fitoterápicos na Atenção Primária em Saúde: percepção dos profissionais. Ciência \& Saúde Coletiva. 23(11), 3735-3744.

Ministério da Saúde. (2012). Práticas Integrativas e Complementares: Plantas Medicinais e Fitoterapia na Atenção Básica. http://bvsms.saude.gov.br/bvs/publicacoes/praticas_integrativas_complementares_plantas_medicinais_cab31.pdf

Nascimento, M. V. N., \& Oliveira, I. F. (2016). As práticas integrativas e complementares grupais e sua inserção nos serviços de saúde da atenção básica. Estudos de Psicologia. 21(3), 272-281, 2016.

Nunes, J. D., \& Maciel, M. V. (2017). A importância da informação do profissional de enfermagem sobre o cuidado no uso das plantas medicinais: uma revisão de literatura. Revista Fitos. 10(4), 375-547.

Pádua, E. M. M. (2019). Metodologia da pesquisa: abordagem teórico-prática. Campinas: Papirus Editora.

Pun, J., Chor, W., \& Zhong, L. (2019). Delivery of patient-centered care in complementary medicine: Insights and evidence from the Chinese medical practitioners and patients in primary care consultations in Hong Kong. Complementary therapies in medicine. 45, $198-204$.

Sá, A. C. M. G. N., Ferreira, E. R. O., Xavier, J. C., \& Alves, C. M. (2018). Contribuições da educação permanente para qualificação da assistência de enfermagem em um hospital público. Rev. bras. ciênc. Saúde. 22(1), 87-94.

Sampaio, L. A., Oliveira, D. R., Kerntopf, M. R., Brito, F. E. J., \& Menezes, I. R. A. (2013). Percepção dos enfermeiros da estratégia saúde da família sobre o uso da fitoterapia. Revista Mineira de Enfermagem. 17(1), 77-85.

Santos, V. P., \& Trindade, L. M. P. (2017). A enfermagem no uso das plantas medicinais e da fitoterapia com ênfase na saúde pública. Revista Científica FacMais. 8(1), 16-34. 
Research, Society and Development, v. 10, n. 13, e427101321593, 2021

(CC BY 4.0) | ISSN 2525-3409 | DOI: http://dx.doi.org/10.33448/rsd-v10i13.21593

Sharp, D., Lorenc, A., Feder, G., Little, P., Hollinghurst, S., Mercer, S., \& MacPherson, H. (2018). 'Trying to put a square peg into a round hole': a qualitative study of healthcare professionals' views of integrating complementary medicine into primary care for musculoskeletal and mental health comorbidity. BMC complementary and alternative medicine. 18(1), 1-14.

Silva Jr, J. N. B., Guedes, H. C. S., Silva, V. C. L., Ferreira, M. G. N., Santos, A. F., \& Madruga, M. D. D. (2017). A enfermagem e a utilização de plantas medicinais no âmbito da atenção básica. Revista de Ciências da Saúde Nova Esperança. 15(3), 61-68.

SILVA, N. C. S., Malaquias, A. V., Bessa, D. H. S., \& Barros, R. M. S. (2017). A utilização de plantas medicinais e fitoterápicos em prol da saúde. Única Cadernos Acadêmicos. 3(3), 1-5.

Van der Watt, A. S. J., Nortje, G., Kola, L., Appiah-Poku, J., Othieno, C., Harris, B., Oladeji, B. B., Esan, O., Makanjuola, V., Price, L. N., Seedat, S., \& Oye, G. (2017). Collaboration between biomedical and complementary and alternative care providers: barriers and pathways. Qual Health Res. 27(14), 2177-2188.

Wardle, J. L., Sibbritt, D. W., \& Adams, J. (2018). Primary care practitioner perceptions and attitudes of complementary medicine: a content analysis of freetext responses from a survey of non-metropolitan Australian general practitioners. Primary health care research \& development. 19(3), 246-255. 